The authors highlight several limitations of the trial and comment that the results require confirmation in a randomized study.

Original article Bobrow BJ et al. (2008) Minimally interrupted cardiac resuscitation by emergency medical services for outof-hospital cardiac arrest. JAMA 299: 1158-1165

\section{Transplanted tissue-engineered smooth-muscle sheets promote neovascularization}

Surgical and percutaneous interventions for peripheral arterial disease only treat focal lesions, and do not reduce atherosclerosis, causing restenosis, and disease recurrence at other sites. The induction of angiogenesis is, therefore, desirable in the treatment of this disease. Some studies have suggested that therapeutic angiogenesis can be achieved through the administration of specific growth factors or the transplantation of isolated cells. However, these strategies have shown limited success; effective revascularization might require administration of multiple growth factors. Outcomes could be improved by transplantation of cell sheets, which can be applied to target lesions without becoming isolated from neighboring cells, and which might secrete more growth factors than isolated cells.

In a Japanese study, Hobo and colleagues investigated the effect of transplanted tissueengineered cell sheets on blood-vessel formation and functional recovery in athymic rats with limb ischemia. The rats were divided into three groups and received either xenografts of human smooth-muscle-cell sheet or fibroblast-cell sheet, or were untreated controls. All rats were monitored for 21 days. Significant improvements in perfusion were observed in the treatment groups compared with the control group throughout the study period. Smooth-muscle cells achieved the greatest improvement and were associated with development of mature capillary networks. In vitro, both cell types secreted proangiogenic growth factors that stimulate blood vessel formation, with the greatest potency observed in smooth-muscle cells.

The authors explain that the transplanted smooth-muscle cells induced neovascularization by localized secretion of proangiogenic growth factors and by migration into ischemic tissues, where they became incorporated into new blood vessels.

Original article Hobo Ket al. (2008) Therapeutic angiogenesis using tissue engineered human smooth muscle cell sheets. Arterioscler Thromb Vasc Biol 28: 637-643

\section{High lipoprotein(a) levels independently predict risk of coronary heart disease}

Previous studies have indicated an association between circulating lipoprotein(a) levels and coronary heart disease (CHD). Now, Bennet et al. have conducted a large-scale study to assess the nature of this link and how it is affected by established cardiovascular risk factors. They conclude that lipoprotein(a) levels have an independent and almost continuous association with $\mathrm{CHD}$ and could prove a useful biomarker for risk of future CHD.

The authors screened a prospectively registered cohort of 18,569 individuals included in the Reykjavik study who had no history of myocardial infarction. Within the subgroup of patients with baseline lipoprotein(a) measurements, individuals who had experienced a first-ever nonfatal myocardial infarction or CHD-related death during follow-up were each paired for analysis with one or two matched controls who did not develop CHD.

Logarithmically transformed baseline lipoprotein(a) levels were higher in patients who developed CHD $(n=2,047)$ than in controls $(n=3,921)$ and changed little after approximately 12 years in the 372 patients for whom a second measurement was available. Log baseline lipoprotein(a) levels and established and emerging cardiovascular risk factors (e.g. albumin levels and blood pressure) did not correlate. Individuals with baseline lipoprotein(a) levels in the top third of the recorded values were more likely to develop CHD than were individuals in the bottom third (odds ratio after adjustment for age, sex and calendar year of recruitment $1.60,95 \% \mathrm{Cl} 1.38-1.85)$. Odds ratios for CHD increased continuously with increasing fifths of baseline lipoprotein(a) level.

Original article Bennet A et al. (2008) Lipoprotein(a) levels and risk of future coronary heart disease: large-scale prospective data. Arch Intern Med 168: 598-608 